\title{
Cataract surgery rates
}

To the Editor: Thank you for publishing the article by Lecuona and Cook on the important topic of cataract surgical rates (CSR) in the RSA. ${ }^{1}$ Indeed, the difficulty in increasing the CSR to the recommended annual target of 2000 (to eliminate blindness from cataract) is exactly what others in Africa have experienced. Even in some very well-served districts with model programmes and high cataract surgical coverage, we find that a CSR of about 1500 or less is all that we manage to achieve. With this in mind, we developed a model to estimate the incidence of cataract (blinding as well as visually impairing) from population-based, age-specific cataract prevalence data. ${ }^{2}$ The incidence rates can be used to predict what the CSR would need to be to eliminate blinding or visually impairing cataract. Having now applied this model to over 20 population-based surveys from Africa, we believe that the target of 2000 to eliminate cataract blindness was simply too high. It was based on estimates of total blindness in Africa that were probably not representative because they were extrapolated from areas that had particularly high levels of blindness, sometimes from focal diseases such as trachoma and onchocerciasis.

The good news is that we can and should put more emphasis on offering cataract surgery to people when most people want surgery, which is before their vision is reduced to blindness. ${ }^{3}$ Of course, it is ever more important that the quality of surgery be very high, and the authors note the potential for high-volume, high-quality surgery. The issues that they document still apply if we are to restore good vision to everyone suffering from cataract: sufficient access to theatre, good management support, better use of scarce resources, and commitment from hospitals and health managers.

\section{Susan Lewallen}

Kilimanjaro Centre for Community Ophthalmology

Moshi

Tanzania

slewallen@kcco.net

1. Lecuona K, Cook C. South Africass cataract surgery rates - why are we not meeting our targets? S Af Med J; 101:510-512.

2. Lewallen S, Williams T, Dray A, et al. Estimating incidence of vision-reducing cataract in Africa: a new model with implications for program targets. Arch Ophthalmol 2010;128:1584-1589.

3. Chibuga E, Massae P, Geneau R, Mahande M, Lewallen S, Courtright P. Acceptance of cataract surgery in a cohort of Tanzanians with operable cataract. Eye 2008;22(6):830-833.

Drs Lecuona and Cook respond: We thank Dr Lewallen for her response to our article. Dr Lewallen and colleagues have developed a model to estimate the incidence of vision-reducing cataract in specific health districts in Africa, on the basis of population-based rapid assessment surveys in those districts, and from that to calculate the cataract surgical rates (CSRs) that are needed to eliminate blindness and visual impairment owing to cataract. ${ }^{1}$ This model will be of great benefit in planning cataract surgical services. The good news, as Dr Lewallen points out, is that a figure of 2000 per million population per year to eliminate blindness from cataract may be too high in most health districts. However, as a middle-income country, South Africa is unique in sub-Saharan Africa in having more demand than capacity for surgery, with waiting lists for surgery, and with patients with visual impairment and severe visual impairment also requesting surgery. Given this demand greater than capacity, it would seem reasonable for our provinces to maintain 2000 as an achievable and realistic CSR target. 